\title{
Comprehensive Segmentation of Cine Cardiac MR Images
}

\author{
Maxim Fradkin ${ }^{1}$, Cybèle Ciofolo ${ }^{1}$, Benoit Mory ${ }^{1}$, Gilion Hautvast ${ }^{2}$, \\ and Marcel Breeuwer ${ }^{2}$ \\ ${ }^{1}$ Medisys Research Lab, Philips Healthcare, Suresnes, France \\ ${ }^{2}$ Healthcare Informatics, Philips Healthcare, Best, The Netherlands
}

\begin{abstract}
A typical Cardiac Magnetic Resonance (CMR) examination includes acquisition of a sequence of short-axis (SA) and long-axis (LA) images covering the cardiac cycle. Quantitative analysis of the heart function requires segmentation of the left ventricle (LV) SA images, while segmented LA views allow more accurate estimation of the basal slice and can be used for slice registration. Since manual segmentation of CMR images is very tedious and time-consuming, its automation is highly required. In this paper, we propose a fully automatic $2 \mathrm{D}$ method for segmenting LV consecutively in LA and SA images. The approach was validated on 35 patients giving mean segmentation error smaller than one pixel, both for LA and SA, and accurate LV volume measurements.
\end{abstract}

\section{Introduction}

A typical Cardiac Magnetic Resonance (CMR) examination includes acquisition of a sequence of short-axis (SA) and long-axis (LA) images covering the cardiac cycle. Quantitative analysis of the heart function (ejection fraction, myocardial thickness and thickening, etc.) requires segmentation of the left ventricle (LV) SA images. LA views, in addition, allow more reliable LV shape estimation, especially near the apex and the valve plane. The later can be used for the accurate and reproducible determination of the basal SA slice, which is known to be one of the major factors of interobserver variability in LV measurements 12 . Automatic multi-view segmentation methods, enabling to obtain highly reproducible LV measurements [3], are thus highly desirable.

Many publications propose automatic or semi-automatic methods for segmenting the LV in SA CMR images 415/6/7/8] or in multiple views 901011], to mention a few. Since SA CMR images, acquired over multiple breath-holds, are often misregistered due to patient motion or inconsistent respiration, 3D segmentation and analysis methods require a registration preprocessing step 12 1314 15 16]. Alternatively to image-based alignment, one can use for this purpose LV contours extracted from multiple views [17]. In contrast to [3], where LA contours had to be drawn manually to initialize SA segmentation, we propose a fully automatic method following similar workflow.

The paper is organized as follows: Section 2 gives a concise description of the proposed method; in Section 3 validation results are presented and discussed; conclusions are given in Section 4 .

D. Metaxas et al. (Eds.): MICCAI 2008, Part I, LNCS 5241, pp. 178 185, 2008.

(C) Springer-Verlag Berlin Heidelberg 2008 


\section{Method}

Among 2D segmentation methods, explicit active contours [18 remain popular, since the underlying discrete contour representation is sufficient to capture the fairly smooth shape of the LV. However, this representation often produces slowly converging algorithms that are quite sensitive to initial conditions and parameterization. While there are various solutions to tackle those issues within the original framework, an interesting alternative representation using B-splines has been proposed in [19. This continuous representation is compact and allows the semi-local control of smooth curves. Those advantages have motivated the design of our spline-based deformable template of the LV.

Most active contour methods are based on the minimization of an objective criterion and tend to be locally trapped by spurious image features during the optimization process. The greedy optimization framework 20] used in this paper offers a valuable compromise between computational complexity, robustness and flexibility. Not only it gives in most cases an acceptable solution by avoiding local minima, but also allows easy incorporation of dissimilar image constraints and prior knowledge (e.g., both differentiable and non-differentiable). We follow a two-stage approach: First, we robustly detect LV position on LA view(s) using standard image acquisition geometry and rigid template deformation. The precise delineation of the LV is done by deforming the template locally, through greedy optimization. Second, the segmented LA contours are used for initialization of deformable templates in the relevant SA slices, which are then adjusted using the same, as for LA, optimization paradigm.

\subsection{Deformable Template Geometry}

Our parametric template relies on interpolating splines controlled by as few nodes as possible. More specifically, the myocardium is modeled as a closed ribbon structure, composed of an imaginary centerline (dashed in Fig. 1) and a variable width. Both the centerline $\boldsymbol{C}(s)=[x(s), y(s)]$ and the ribbon width $w(s)$ are continuous spline interpolations of a discrete set of $\left\{p_{k}=\left(x_{k}, y_{k}, w_{k}\right)\right\}$ samples defined at each node. Among the advantages of this compact representation is the natural coupling between the endocardium and the epicardium (the inside and outside contours). We also define two enclosed regions $\mathcal{M}$ and $\mathcal{B}$, corresponding respectively to the myocardium and blood pool areas, as shown in Fig. 1

\subsection{Minimization Criterion}

The optimal solution of our segmentation problem is the set of template parameters minimizing a criterion that expresses the goodness of fit between the geometrical model and the image evidence, given some a priori knowledge. In the remainder, $I$ is the image and the $\lambda_{i}$ 's are scalar weights balancing the various terms. Let $\boldsymbol{p}=\left\{p_{k}=\left(x_{k}, y_{k}, w_{k}\right)^{T}, k \in \llbracket 1, N \rrbracket\right\}$ be our parametric model. The problem can now be formalized as follows: 

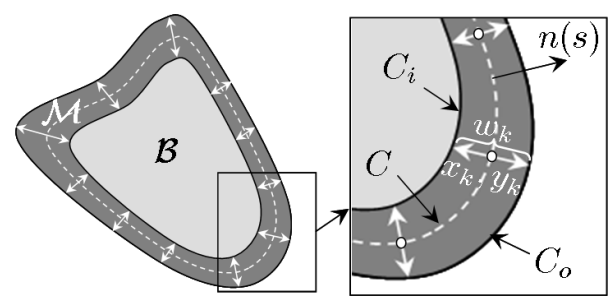

$\boldsymbol{C}_{\boldsymbol{i}}(s)=\boldsymbol{C}(s)-0.5 w(s) \boldsymbol{n}(s)$

$\boldsymbol{C}_{\boldsymbol{o}}(s)=\boldsymbol{C}(s)+0.5 w(s) \boldsymbol{n}(s)$

Fig. 1. Template geometry (left); Detailed zoom (center)

$$
\min _{\boldsymbol{p}}\{F(\boldsymbol{p}, I)=\underbrace{F_{s}(\boldsymbol{C}, w)}_{\text {shape }}+\underbrace{F_{c}\left(\boldsymbol{C}_{\boldsymbol{i}}, \boldsymbol{C}_{\boldsymbol{o}}, I\right)}_{\text {contour }}+\underbrace{F_{r}(\mathcal{M}, \mathcal{B}, I)}_{\text {region }}\}
$$

Shape: Shape constraint is composed of three terms:

$$
F_{s}(\boldsymbol{C})=\lambda_{0} F_{\text {regularity }}(\boldsymbol{C}, w)+\lambda_{1} F_{\text {smoothness }}(\boldsymbol{C})+\lambda_{2} F_{\text {similarity }}(\boldsymbol{C})
$$

The first one regularizes template width:

$$
F_{\text {regularity }}(\boldsymbol{C}, w)=\int_{0}^{1}\left|w^{\prime}(s)\right| d s
$$

The smoothness term controls centerline curvature:

$$
F_{\text {smoothness }}(\boldsymbol{C})=\int_{0}^{1}\left|\kappa(s)-\kappa_{0}\right| d s
$$

where $\kappa(s)$ stands for the curvature of the centerline $\boldsymbol{C}(s)$ and $\kappa_{0}$ is a desired curvature, e.g. setting it to average curvature constrains the contour to be circular (see Sec. 2.5).

The similarity term constraints the template curve shape to be affinely similar with a pre-defined shape $\tilde{\boldsymbol{C}}$. It is defined as an error of the best affine transformation $T$ between the given contour $\boldsymbol{C}$ and a pre-defined contour $\tilde{\boldsymbol{C}}$ :

$$
F_{\text {similarity }}(\boldsymbol{C})=\int_{0}^{1}|\boldsymbol{C}(s)-T(\tilde{\boldsymbol{C}}(s))|^{2} d s
$$

Contour: The contour term stands for the contour contrast, both local (first two terms) and global (last term):

$$
F_{c}\left(\boldsymbol{C}_{\boldsymbol{i}}, \boldsymbol{C}_{\boldsymbol{o}}, I\right)=\lambda_{3} \int_{0}^{1} \nabla I\left(\boldsymbol{C}_{\boldsymbol{i}}(s)\right) \cdot \boldsymbol{n}(s) d s-\lambda_{4} \int_{0}^{1}\left|\nabla I\left(\boldsymbol{C}_{\boldsymbol{o}}(s)\right) \cdot \boldsymbol{n}(s)\right| d s+\lambda_{5}(\bar{m}-\bar{b})
$$

where $\nabla I$ is the image gradient, $n(s)$ contour's normal, $\bar{m}$ and $\bar{b}$ are the average intensities of the regions $\mathcal{M}$ and $\mathcal{B}$, respectively. 
Region: The myocardium and the blood pool gray levels, except for the papillary muscles region, should be homogeneously distributed. Therefore we can define $F_{r}$ using a minimal variance criterion such as:

$$
F_{r}(\mathcal{M}, \mathcal{B}, I)=\frac{\lambda_{6}}{|\mathcal{M}|} \int_{\mathcal{M}}|I(x, y)-\bar{m}|^{2} d x d y+\frac{\lambda_{7}}{|\mathcal{B}|} \int_{\mathcal{B}}|I(x, y)-\bar{b}|^{2} d x d y
$$

where region $\mathcal{M}$ (resp. $\mathcal{B}$ ) has average intensity $\bar{m}$ (resp. $\bar{b}$ ) and area $|\mathcal{M}|$ (resp. $|\mathcal{B}|)$. In order to increase robustness to the expected presence of papillary muscles, which breaks the blood pool homogeneity assumption, a rather effective approach is to remove the contribution of a certain percentage (e.g., 15\%) of the darkest pixels in region $\mathcal{B}$ from the variance computation.

\subsection{Optimization Strategy}

We use a greedy optimization scheme embedded in a coarse-to-fine approach to optimize simultaneously the nodes position and ribbon width. Due to using very few nodes and a global criterion, each optimization step influences an important portion of the model, thus allowing potential jumps over local minima. Unfortunately, casting normals with such a sparse sampling makes the solution dependent on the initial position of the nodes, hence decreasing parameterization invariance. We compensate for this drawback of the original scheme by a systematic sliding of all nodes along the centerline between each iteration.

\subsection{Segmentation of Long Axis Images}

Template creation and initial pose: The initial template should resemble the LV shape, so its centerline can be approximated by an ellipse which axes are aligned with the main axes of the LV; it is slightly bended inside for modeling valve shape (Fig. 2-left). For initialization of LA template, we need to define its position $\left(p_{c}\right)$ and orientation $(\vec{u})$. These parameters can be first obtained in patient coordinates, and then easily transformed into the image ones. When two LA views are available, we reasonably assume (due to standard acquisition planning) that an intersection of the two LA image planes is approximately aligned with the real LA of the heart (Fig. 2-center) and therefore can be taken as $\vec{u}$ (it is taken co-oriented with the $Z$ axis of the SA). The template origin $p_{c}$ is obtained as an intersection of the axis $\vec{u}$ with the middle slice of the SA volume. When only one LA view is available, we align $\vec{u}$ with $Z$ axis of the SA volume, and the template origin is defined as a closest $\vec{u}$ point to the center of SA volume $p_{o}$, since a typical SA acquisition is centered on LV (Fig. 2right).

Template deformation: The deformation is done in two steps: coarse (rigid) and fine (local). At the coarse step, the initial template is affinely optimized using only two minimization terms, namely contour (Eq. 2) and region (Eq. 3), since the shape is implicitly conserved by the transformation. Note also that 

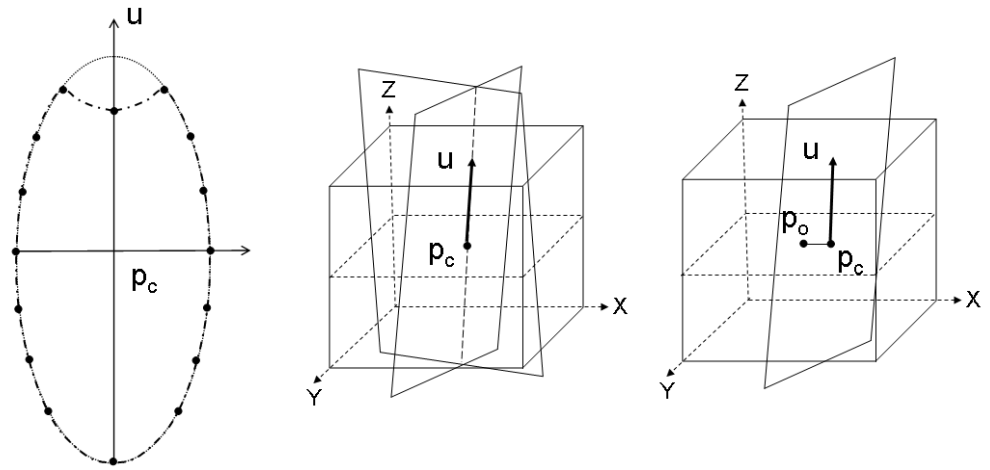

Fig. 2. LA template: centerline as an ellipse with upper bending modeling the valve (left); LA template initial pose using SA volume, represented as a cube, and two LA views, represented as planes (center); Similarly to later, LA template initial pose with SA volume and only one LA view (right)

in the contour term only global contrast contributes at this stage $\left(\lambda_{3}, \lambda_{4} \rightarrow 0\right)$. This step, allowing to robustly detect the LV position, is followed by a local deformation, using all three terms. In the contour constraint, only local contrast is now put to contribution $\left(\lambda_{5} \rightarrow 0\right)$, while in the shape constraint (Eq. 11) only regularity and similarity terms are used $\left(\lambda_{1} \rightarrow 0\right)$. In addition, contour resulting from the coarse step is used as $\tilde{C}$, which preserves certain contour similarity with the initial shape and in particular allows to softly constrain valve concavity.

\subsection{Segmentation of Short Axis Images}

Template creation and initial pose: The SA template centerline can be approximated by a circle. Thus, SA template creation is quite obvious since one only needs to define the template center and radius (Fig. 3-left). Given the LA segmentation results, the LV LA contour(s) intersections are found at each SA slice. If two LA contours are available, the template parameters are found by fitting a best circle through 4 intersection points (Fig. 3 - center), while if only one contour is given, the template center is defined as the middle of the intersection segment and its radius as a half of its length (Fig. 3-right). Note that if no LA images are available, the initialization of SA templates can still be done using robust ring detection algorithm [21.

Template deformation: The SA template deformation follows the same coarseto-fine scheme as the LA. The optimization terms used at coarse step are exactly the same as in Sec. 2.4. At the fine step, the contour and the region terms are again the same as for LA, while the shape constraint comprises only regularity and smoothness terms (in the later one, $\kappa_{0}$ is set to the average contour curvature to impose contour circularity, thus also substituting the similarity term). 

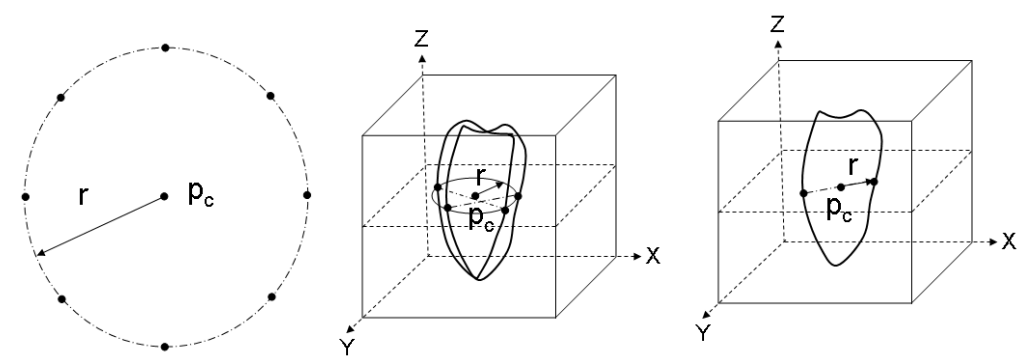

Fig. 3. SA template centerline as a circle (left); SA template initial pose using intersection of two LA contours with a SA volume slice (center); SA template initial pose using intersection of only one LA contour with a SA volume slice (right)

\section{Validation}

Data: The clinical data used for validating our method included 35 patients. SA cine CMR scans comprised 9-14 slices and 15-50 phases; all SA images were $256 \times 256$ in size, covering a field of view ranging from 360x360 mm up to $450 \times 450$ mm. For 5 patients two LA views ( 2 chambers $(\mathrm{CH})$ and $4 \mathrm{CH}$ ) were available, while for the remaining ones only $4 \mathrm{CH}$ LA views were acquired. All LA images were $256 \times 256$ in size, covering a field of view ranging from 350x350 mm up to 460x460 mm. Manual delineations, done by an expert, were available for all enddiastolic (ED) images (325 in total of SA and 40 for LA).

Results: To compare the result of our automatic segmentation method with the manually delineated contours, we measured the mean distance $\left(\varepsilon_{\text {mean }}\right)$, the root-mean-square (RMS) distance $\left(\varepsilon_{r m s}\right)$ and the maximum distance $\left(\varepsilon_{\max }\right)$ between the resulting contours and the manual ones, using method explained in 22. For SA images, mean positioning errors was $1.3 \mathrm{~mm}$ for the endocardium and $1.5 \mathrm{~mm}$ for the epicardium, for a pixel size ranging from $1.4 \mathrm{~mm}$ to $1.8 \mathrm{~mm}$ (see Table 1). For LA images, mean positioning errors was $1.3 \mathrm{~mm}$ for the endocardium and $1.1 \mathrm{~mm}$ for the epicardium, for a pixel size ranging from $1.3 \mathrm{~mm}$ to $1.8 \mathrm{~mm}$. An excellent correlation was found between "manual" and "automatic" volumes $(r=0.90, P<0.001)$, with mean difference $-10 \pm 18 \mathrm{ml}(P=0.002)$. Note that all results were obtained with exactly the same method settings and without any user interaction. An example of segmentation results for one patient (2 LA views and $8 \mathrm{SA}$ slices) is shown in Fig. 4. In our current implementation, the overall segmentation time is around 1 second per image on a $3.2 \mathrm{GHz} \mathrm{PC}$, thus segmentation of a typical image phase $(\mathrm{SA}+\mathrm{LA})$ takes about 10 seconds.

Discussion: LA template initialization proved to be robust even using only one LA image, the same stands for the initialization of SA templates from only one LA segmentation contour. Note also that SA initialization was robust to slices misregistration, since possible initialization inaccuracies were compensated by the rigid deformation. Producing a mean positioning error inferior to one pixel for 
Table 1. Endocardial and epicardial positioning errors compared to manual contours

\begin{tabular}{|l|c|c|c|c|c|c|}
\hline \multicolumn{4}{|c|}{ Endocardial contours } & \multicolumn{3}{c|}{ Epicardial contours } \\
\hline & $\varepsilon_{\text {mean }}(\mathrm{mm})$ & $\varepsilon_{r m s}(\mathrm{~mm})$ & $\varepsilon_{\max }(\mathrm{mm})$ & $\varepsilon_{\text {mean }}(\mathrm{mm})$ & $\varepsilon_{r m s}(\mathrm{~mm})$ & $\varepsilon_{\max }(\mathrm{mm})$ \\
\hline $\mathrm{SA}$ & $\mathbf{1 . 2 7} \pm \mathbf{0 . 5 4}$ & $\mathbf{1 . 5 6} \pm \mathbf{0 . 7 0}$ & $\mathbf{3 . 2 9} \pm \mathbf{1 . 6 2}$ & $\mathbf{1 . 5 6} \pm \mathbf{0 . 8 5}$ & $\mathbf{1 . 9 3} \pm \mathbf{1 . 0 4}$ & $\mathbf{4 . 0 6} \pm \mathbf{2 . 1 8}$ \\
\hline $\mathrm{LA}$ & $\mathbf{1 . 3 2} \pm \mathbf{0 . 4 1}$ & $\mathbf{1 . 6 9} \pm \mathbf{0 . 5 5}$ & $\mathbf{4 . 5 5} \pm \mathbf{1 . 8 4}$ & $\mathbf{1 . 1 3} \pm \mathbf{0 . 4 2}$ & $\mathbf{1 . 5 2} \pm \mathbf{0 . 6 7}$ & $\mathbf{4 . 0 1} \pm \mathbf{2 . 2 7}$ \\
\hline
\end{tabular}
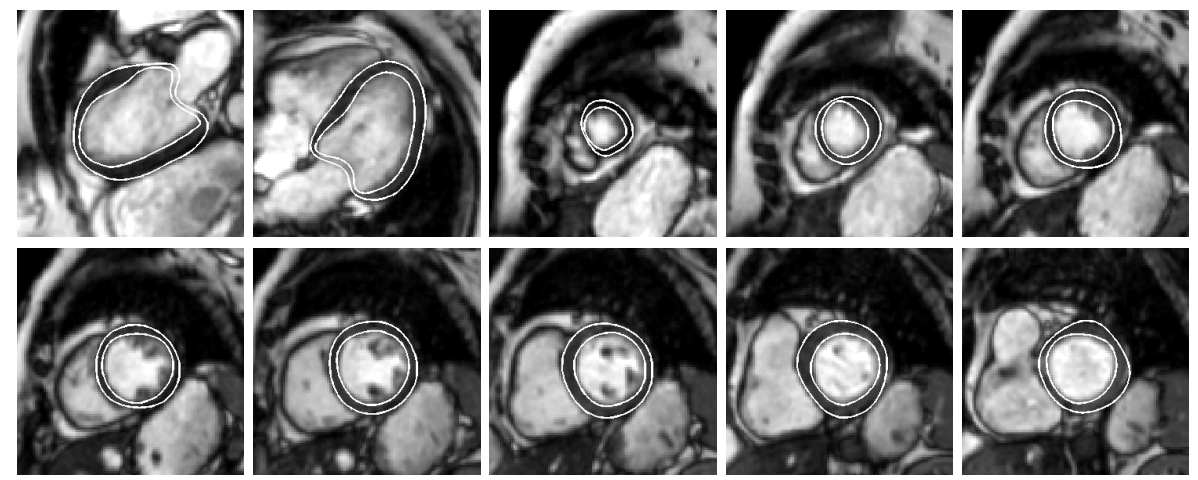

Fig. 4. LV segmentation example for one patient (from left to right, up to bottom): LA $2 \mathrm{CH}$ image; LA 4CH image; consecutive slices of SA volume from apex to valve

both SA and LA images, comparable with inter-expert drawing variability [1], the method proved accurate and robust. We validated the method for ED phase only, since the segmentation of the whole cardiac cycle can be then obtained using automatic contour propagation [22, shown to preserve its accuracy within acceptable ranges while being much faster than phase-by-phase segmentation.

\section{Conclusion}

We developed a fully automatic, robust and accurate method for segmentation of CMR SA and LA acquisitions, enabling easy introduction of prior knowledge and constraints in the optimization framework. The method was extensively validated by comparing the segmentation results with manually drawn endocardial and epicardial contours for 35 patients (325 SA slices and 40 LA images). The positioning error was inferior to one pixel for both SA and LA images and is comparable with interobserver variability. The resulting contours can be used for SA slice alignment, contour propagation and accurate LV parameter estimation.

\section{References}

1. Weaver, A., et al.: Magnitude and causes of interobserver discrepancies in CMR volumme measurements: critical importance of choice of the basal slice. Journal of Cardiovascular Magnetic Resonance 1, 82-83 (2006) 
2. Anderson, J., et al.: Normal cardiac magnetic resonance measurements and interobserver discrepancies in volumes and mass using the papillary muscle inclusion method. The Open General and Internal Medicine Journal 1, 6-12 (2007)

3. van Geuns, R., et al.: Automatic quantitative left ventricular analysis of cine MR images by using threedimensional information for contour detection. Radiology 240(1), 215-221 (2006)

4. Mitchell, S.C., et al.: Multistage hybrid active appearance model matching: segmentation of left and right ventricles in cardiac MR images. IEEE Trans. Med. Imag. 20(5), 415-423 (2001)

5. Kaus, M.R., et al.: Automated segmentation of the left ventricle in cardiac MRI. Medical Image Analysis 8(3), 245-254 (2004)

6. Niessen, W.J., et al.: Geodesic deformable models for medical image analysis. IEEE Trans. Med. Imag. 17(4), 634-641 (1998)

7. Paragios, N.: A variational approach for the segmentation of the left ventricle in cardiac image analysis. Int. J. Comp. Vision 50(3), 345-362 (2002)

8. Jolly, M.P.: Automatic segmentation of the left ventricle in cardiac MR and CT images. Int. J. Comp. Vision 70(2), 151-163 (2006)

9. Lelieveldt, B., et al.: Multi-view active appearance models for consistent segmentation of multiple standard views: application to long- and short-axis cardiac MR images. In: Int. Congress Series, vol. 1256, pp. 1141-1146 (2003)

10. Lotjonen, J., et al.: Statistical shape model of atria, ventricles and epicardium from short- and long-axis MR images. Medical Image Analysis (8), 371-386 (2004)

11. van Assen, H., et al.: SPASM: A 3D-ASM for segmentation of sparse and arbitrarily oriented cardiac MRI data. Medical Image Analysis 10, 286-303 (2006)

12. Moore, J., et al.: A high resolution dynamic heart model based on averaged MRI data. In: Ellis, R.E., Peters, T.M. (eds.) MICCAI 2003. LNCS, vol. 2878, pp. 549 555. Springer, Heidelberg (2003)

13. Lotjonen, J., et al.: Correction of movement artifacts from 4-d cardiac short- and long-axis MR data. In: Barillot, C., Haynor, D.R., Hellier, P. (eds.) MICCAI 2004. LNCS, vol. 3217, pp. 405-412. Springer, Heidelberg (2004)

14. Perperidis, D., et al.: Statistical shape model of atria, ventricles and epicardium from short- and long-axis MR images. Medical Image Analysis 9(8), 441-456 (2005)

15. Hautvast, G., Cocosco, C., Kedenburg, G., Breeuwer, M.: Alignment of short axis and long axis cine cardiac MR images. In: CARS 2006, vol. 1, pp. 59-60 (2006)

16. Slomka, P.J., et al.: Automated registration of multiple single breath-hold cardiac MRI images. Intl. Soc. Mag. Reson. Med. 14, 1207 (2006)

17. Li, T., Denney, T.: Registration of short and long-axis images in cine cardiac MRI. Intl. Soc. Mag. Reson. Med. 14, 1208 (2006)

18. Kass, M., Witkin, A., Terzopoulos, D.: Snakes: active contour models. Int. J. Comp. Vision 1(4), 321-331 (1987)

19. Menet, S., Saint-Marc, P., Medioni, G.B.: BSnakes: implementation and application to stereo. In: Image Understanding Workshop, Pittsburgh, PA, pp. 720-726 (1990)

20. Williams, D.J., Shah, M.: A fast algorithm for active contours and curvature estimation. CVGIP: Image Understanding 55(1), 14-26 (1992)

21. Breeuwer, M., Hautvast, G., Mory, B., Fradkin, M.: Automatic segmentation of short-axis cine cardiac magnetic resonance. In: CARS 2008 (2008)

22. Hautvast, G., et al.: Automatic contour propagation in cine cardiac magnetic resonance images. IEEE Trans. Med. Imag. 25(11), 1472-1482 (2006) 